\title{
QUANDO SUSPEITAR DE CARDIOPATIA CONGÊNITA NO RECÉM-NASCIDO
}

\author{
WHEN CONGENITAL HEART DISEASE SHOULD BE SUSPECTED IN THE NEWBORN
}

Fernando Amaral' ${ }^{1}$; João Antonio Granzotti²; Paulo Henrique Manso ${ }^{3}$ \& Luisa Sajovic de Conti ${ }^{4}$

\begin{abstract}
${ }^{1}$ Faculdade de Medicina da UNAERP. Médico Assistente do HCFMRP-USP. ${ }^{2}$ Setor de Cardiologia Pediátrica do Departamento de Puericultura e Pediatria da Faculdade de Medicina de Ribeirão Preto - USP. ${ }^{3}$ Médico Assistente do HCFMRP-USP. ${ }^{4}$ Graduanda da Faculdade de Medicina - UNAERP.

Correspondencia: Dr. Fernando Amaral. Setor de Cardiologia Pediátrica. Departamento de Puericultura e Pediatria. Hospital das Clínicas da Faculdade de Medicina de Ribeirão Preto-USP. Campus Universitário 14048-900. Ribeirão Preto - SP. e-mail: ftvamaral@bol.com.br
\end{abstract}

Amaral F; Granzotti JA; Manso PH \& Conti LS. Quando suspeitar de cardiopatia congênita no recém-nascido. Medicina, Ribeirão Preto, 35: 192-197, abr./jun. 2002.

RESUMO: Artigo de revisão sobre as principais manifestações clínicas das cardiopatias congênitas, que se manifestam no período neonatal. A partir da presença de dados clínicos, como sopro cardíaco, cianose, taquipnéia e arritmia, são discutidos os principais elementos clínicos associados, assim como as entidades mais provavelmente a eles relacionadas. Informações sobre diagnóstico e terapêutica são também sumariamente apresentadas.

UNITERMOS: Cardiopatias Congênitas. Recém-nascido. Sopros Cardíacos.

\section{INTRODUÇÃO}

O período neonatal para o paciente portador de cardiopatia congênita pode ser crítico, fundamentalmente, devido a dois fatores: a gravidade de alguns defeitos comumente presentes e as modificações fisiológicas que normalmente ocorrem nessa fase. Sendo assim, acreditamos que a atenção primária para com esse grupo de crianças deva ser feita de maneira muito cuidadosa, objetivando reconhecer a cardiopatia precocemente. Tentaremos abordar esse tema, procurando valorizar, sobretudo, o exame clínico da criança, técnica essa que acreditamos possa ser bem desenvolvida pelo pediatra e pelo cardiologista.

A suspeita clínica de cardiopatia congênita no período neonatal pode ser levantada pela presença de quatro achados principais: Sopro cardíaco, cianose, taquipnéia e arritmia cardíaca.

\section{MANIFESTAÇÕES CLÍNICAS}

\subsection{Sopro cardíaco}

É um achado freqüente no recém-nascido (RN) e a questão inicial que se apresenta é: o sopro é inocente ou patológico? Sabemos que o sopro inocente é achado extremamente comum em crianças normais e seu diagnóstico é exclusivamente clínico, sem necessidade de exames complementares, exceto naqueles casos duvidosos ${ }^{1,2,3}$. Entretanto, deve ser enfatizado que o sopro inocente no neonato não é tão frequiente quanto em outras faixas etárias, sendo aconselhável uma investigação clínica cuidadosa ou reavaliação freqüente dentro das primeiras semanas de vida. Um tipo de sopro inocente, comumente encontrado nesse período, é aquele originado pela desproporção que normalmente existe entre a artéria 
pulmonar central e seus ramos, sendo audível bilateralmente, inclusive no dorso e com tendência ao desaparecimento precoce ${ }^{4}$. De forma semelhante ao encontrado em outras faixas etárias, o sopro de Still, audível no $3^{\circ}$ e no $4^{\circ}$ espaço intercostal esquerdo, o sopro no foco pulmonar e o sopro supraclavicular são modalidades de sopro inocente que também podem ser detectados no RN, porém com menor freqüência. Por outro lado, a caracterização do sopro patológico deve ser baseada em três dados: na maioria dos casos, esses sopros são rudes, quase sempre sistólicos e usualmente localizados. São muitas as causas desse ruído patológico, sendo as mais freqüentes a comunicação interventricular (CIV), a persistência do canal arterial (PCA), a estenose da valva pulmonar (EP) e a estenose da valva aórtica (EAo). A insuficiência da valva tricúspide, comumente associada com o período de hipertensão pulmonar transitória do RN, pode apresentar-se com sopro sistólico no foco tricúspide, e tende a desaparecer progressivamente. É importante enfatizar que, mesmo no recém-nascido, existe um grau relativamente alto de especificidade entre o achado semiológico à ausculta e o tipo de defeito cardíaco, encontrado naquelas cardiopatias consideradas "simples", geralmente manifestando-se como lesão única. Entre as cardiopatias complexas, entretanto, é comum, porém não obrigatória, a presença de um sopro sistólico com menor grau de especificidade diagnóstica (Tabela I). Na abordagem desses pacientes com sopro, acreditamos que três conceitos fundamentais devam ser discutidos.

- Sopro rude significa cardiopatia grave?

- Neonato com sopro e assintomático: bom prognóstico?

- Neonato sem sopro: existe cardiopatia?

O desdobramento desses três conceitos, certamente, permite ampla discussão, em virtude da grande variedade de cardiopatias passíveis de serem detectadas no período neonatal. Procuraremos mostrar aqui as causas mais freqüentes de cardiopatias, envolvidas nesses tópicos, procurando oferecer um aspecto prático ao tema (Tabela II).

\section{$\left.1^{\circ}\right)$ Sopro rude significa cardiopatia grave?}

Não necessariamente. Um exemplo muito comum é a criança nascida com CIV, que classicamente apresenta sopro sistólico rude e é assintomática, quando o diâmetro do orifício é pequeno ${ }^{5}$. Outras situações são as estenoses das valvas pulmonar ${ }^{6} \mathrm{e}$ aórtica de grau moderado, que também podem apre-
Tabeh I: Principais causas de sopro no neo nato

- Comuricação Interventricular

- Persistência do Canal Arterial

Estenose da Valva Pulmonar

Estenose da Valva Aórtica

Sopro Inocente

- Insticiêrcia Tricúspide Funcional

- C ardiopatias Complexas Variadas

Tabeh II: Sopro Cardíaco wo Neonato - Conceitos

Sopro inocente: menos freqüente que em outras faxas etárias

Sopro nude: não indica necessariamente cardiopatia gave

C ardiopatia grave pode se marifestar com sopro sem sintomas

Ausência de sopro não exclui cardiopatia grave

sentar sopro bem audível e com pouca ou nenhuma repercussão clínica. Freqüentemente, a avaliação clínica cardiológica oferece a possibilidade de, somente com recursos semiológicos, estabelecer o diagnóstico da anomalia. Rotineiramente empregados, o eletrocardiograma e a radiografia simples do tórax proporcionam ajuda considerável na elaboração do diagnóstico, que, na grande maioria dos casos, será confirmado pelo ecocardiograma.

\section{$2^{\circ}$ ) Neonato com sopro e assintomático: bom prognostico?}

Nem sempre. Essa é uma situação bastante peculiar, que requer avaliação clínica detalhada e compreensão precisa da fisiologia cardiovascular neonatal. Pacientes portadores de cardiopatias congênitas com hiperfluxo pulmonar (CIV, CIA, PCA e defeito do septo atrioventricular, entre outras) constituem um grupo importante. No período neonatal, quando os defeitos são grandes e a resistência vascular pulmonar é alta, as crianças costumam ser totalmente assintomáticas. Entretanto, quando a resistência pulmonar diminui, é comum o aparecimento de sintomas progressivos, do tipo dispnéia e sudorese, podendo evoluir para insuficiência cardíaca. Outro exemplo são 
crianças nascidas com coarctação da aorta $^{8}$ com canal arterial patente. Quando o canal se fecha espontaneamente, geralmente no final da $1^{\mathrm{a}}$ semana ou na $2^{\text {a }}$ semana de vida, os sintomas aparecem, podendo ocorrer insuficiência cardíaca grave. Pacientes portadores de estenose aórtica valvar grave podem, também, se apresentar totalmente assintomáticos ao nascer e evoluir rapidamente para quadro de insuficiência cardíaca grave, necessitando intervenção terapêutica invasiva.

Nas situações acima citadas, é fundamental o estabelecimento de um diagnóstico definitivo, geralmente não invasivo, de maneira precoce, que permita prever a possível evolução clínica da criança.

\section{$\left.3^{\circ}\right)$ Neonato sem sopro: existe cardiopatia?}

É possível. Infelizmente, esse ainda é um motivo freqüente para se excluir cardiopatia grave num RN. Dependendo do mecanismo fisiopatológico envolvido, sobretudo nas cardiopatias complexas, o sopro pode estar ausente e a suspeita de cardiopatia deverá ser baseada na presença de outros dados clínicos. Algumas entidades podem se apresentar sem sopro ou, às vezes, com sopro discreto, não devidamente valorizado pelo clínico. Citamos, como exemplos, a coarctação da aorta (palpe os pulsos femorais e avalie a pressão arterial para o diagnóstico), a transposição dos grandes vasos (a $2^{\mathrm{a}}$ bulha está quase sempre bastante hiperfonética e pode ajudar no diagnóstico), a tetralogia de Fallot grave (observe a cianose, analise a $2^{\mathrm{a}}$ bulha e lembre-se que na T. Fallot grave, o sopro pode ser discreto ou mesmo ausente) além do ventrículo único e das atresias pulmonar e tricúspide. Os dados semiológicos, associados ao ECG e radiografia simples do tórax permitem que, na maioria dos casos, chegue-se a um diagnóstico, pelo menos, aproximado da doença, que usualmente será confirmada pelo ecocardiograma.

\subsection{Cianose}

Procurando ser prudentes, acreditamos que a presença de cardiopatia deva sempre ser suspeitada no RN cianótico, independente do quadro clínico e, principalmente, se essa cianose não melhorar rapidamente com a inalação de $0_{2}$. Obviamente, é importante afastar a possibilidade de obstrução das vias aéreas, feita com segurança pelo neonatologista, assim como a presença de hipertensão pulmonar transitória do RN , esta última bastante freqüente e facilmente confundível com cardiopatia cianótica. Uma pista importante para o diagnóstico diferencial é que os pacientes com hipertensão pulmonar transitória costumam ter (porém nem sempre) uma $2^{\mathrm{a}}$ bulha cardíaca, nitidamente desdobrada nos focos pulmonar e tricúspide, diferente das cardiopatias congênitas, cianóticas, complexas, que costumam apresentar $2^{\mathrm{a}}$ bulha única e hiperfonética, como no caso da transposição dos grandes vasos, a cardiopatia cianótica mais comum no período neonatal. O diagnóstico clínico, nesses casos, costuma ser difícil, mesmo com a ajuda do ECG e radiografia de tórax, que, entretanto, devem ser realizados de rotina. Um aspecto importante que deve ser salientado é a necessidade de confirmação diagnóstica rápida nos pacientes cianóticos, independentemente do quadro clínico. Crianças portadoras de cardiopatia cianótica canal-dependente, (circulação pulmonar depende da persistência do canal arterial para sua perfusão), podem estar aparentemente bem pela presença de uma PCA, que tende a se fechar espontaneamente e de maneira rápida. Como exemplos, temos a atresia da valva pulmonar, a tetralogia de Fallot de má anatomia e a atresia da valva tricúspide como as mais frequientes. Nesses casos, é importante a disponibilidade de prostaglandina $\mathrm{E}$ a ser administrada endovenosamente para a manutenção da patência do canal arterial, procurando estabilizar hemodinamicamente a criança $\mathrm{a}^{10,11}$. (Tabela III).

Tabe h III: Causas cardiacas mais freqüentes de cianose ne ortatal

Transposição dos gandes vasos

Tetralogia de $\mathrm{F}$ allot

Atresia da valva pulmonar

Atresia da valva tricúspide

\subsection{Taquipnéia}

O RN com quadro de taquipnéia/dispnéia apresenta urna situação que deve ser avaliada de maneira bastante cuidadosa, em virtude da possibilidade de cardiopatia. Inicialmente, é importante afastar a presença de um quadro de taquipnéia transitória, entidade frequiente na prática diária. Uma informação particularmente útil, nesses casos, é que a maioria dos pacientes com taquipnéia secundária a uma cardiopatia costuma apresentar alterações semiológicas específi- 
cas, como sopro, alterações nas bulhas cardíacas, anormalidades nos pulsos arteriais e presença de ruídos acessórios corno o click, ruído esse freqüentemente confundido com desdobramento de bulha. É fundamental a investigação imediata desses pacientes com ajuda do ECG e radiografia de tórax. O diagnóstico é confirmado pelo ecocardiograma na grande maioria dos casos, sendo raramente necessário o estudo hemodinâmico. Um aspecto fundamental a ser considerado é que a manifestação de taquipnéia pode ser parte de um quadro de insuficiência cardíaca, de gravidade variável, em função da severidade do defeito congênito presente. Algumas situações devem ser lembradas: a coarctação da aorta ${ }^{8}$ é a causa mais comum de insuficiência cardíaca no neonato, com diagnóstico freqüentemente suspeitado pela ausência de pulsos femorais. O quadro pode ser bastante grave, necessitando tratamento medicamentoso e intervenção cirúrgica imediata ou, mais raramente empregada, angioplastia com cateter-balão. A estenose aórtica grave do $\mathbf{R N}^{7}$ é relativamente freqüente, pode ser suspeitada pelo sopro rude e pulsos com amplitude diminuída, e costuma apresentar-se com quadro de falência cardíaca grave, nos primeiros dias de vida. $\mathrm{O}$ tratamento clínico instituído (diuréticos, aminas) costuma ser ineficaz, sendo necessário procedimento invasivo, geralmente, cateterismo intervencionista (dilatação da valva aórtica) como medida vital. A síndrome da hipoplasia do coração esquerdo é doença extremamente grave e complexa, que costuma manifestar-se nas primeiras horas de vida com quadro de falência cardíaca grave, progressiva, evoluindo para choque cardiogênico geralmente de caráter irreversível ${ }^{12}$. A terapêutica clínica deve ser instituída, porém, geralmente é ineficaz. Apesar de recursos cirúrgicos estarem disponíveis, alguns centros, em virtude da alta complexidade da doença, preferem não intervir nesses casos. Algumas cardiopatias cianóticas complexas podem se manifestar precocemente, com quadro de insuficiência cardíaca e cianose, como o tronco arterial comum (truncus), a drenagem venosa anômala total das veias pulmonares, a atresia da valva tricúspide e o ventrículo único, as duas últimas, quando ocorrem com ausência de estenose pulmonar. Esses defeitos podem ser diagnosticados pelo ecocardiograma e requerem sempre intervenção cirúrgica paliativa ou corretiva a curto prazo (Tabela IV).

\subsection{Arritmia}

$\mathrm{Na}$ abordagem inicial do RN com arritmia, é importante lembrar a necessidade de se excluir a pre-
Tabela IV: Causas cardiacas mais freqüentes de taquidisp né ia neo natal

C oarctação da Aorta

Persistência do Canal Arterial no Prematuro

Estenose Valvar Aóttica Grave

Sirdrome Hipoplásica do Coração Escuerdo

Tronco Arterial Comum

C onexão Venosa Anômala Total das Veias Pulmonar es

Atresia Tricúspide e Ventrículo Úrico sem Estenose pulmonar

sença de um defeito estrutural congênito associado, que pode ser ou não responsável pela arritmia. Os distúrbios do ritmo cardíaco podem ser detectados incidentalmente, quando da avaliação neonatal rotineira ou, mais raramente, em situação de urgência, com ou sem repercussão clínica. Citaremos as mais freqüentes.

EXTRÁ-SISTOLIA: nesses casos, nota-se, na ausculta, uma irregularidade do ritmo cardíaco, em paciente usualmente sem evidência de cardiopatia. É importante lembrar a possibilidade da ocorrência de arritmia sinusal (respiratória), entidade comum em crianças normais e que pode ser confundida com extra-sistolia. O diagnóstico, nessa situação, é estabelecido pelo eletrocardiograma convencional. As formas mais comumente encontradas no neonato são as extra-sístoles supraventriculares, consideradas benignas e que podem ocorrer em até $20 \%$ dos prematuros, nos primeiros dias de vida, sem necessitar terapêutica Extra-sístoles ventriculares tendem a ocorrer raramente e, excepcionalmente, podem necessitar terapêutica medicamentosa ${ }^{14}$.

BLOQUEIO ATRIOVENTRICULAR TOTAL CONGÊNITO: pode estar ou não associado a um defeito estrutural ${ }^{15}$. A suspeita diagnóstica é baseada na presença de frequiência cardíaca anormalmente baixa para a idade do paciente, geralmente na faixa de 50 a 70 BPM, devendo ser lembrada sua possível detecção ainda no período fetal. Na maioria dos casos, os pacientes encontram-se clinicamente estáveis do ponto de vista cardiovascular, porém, em algumas situações, quando a frequiência é muito baixa, usualmente menor que $50 \mathrm{BPM}$, pode ocorrer quadro de descompensação cardíaca grave, necessitando intervenção imediata com marca-passo. O diagnóstico é 
confirmado pelo eletrocardiograma convencional e a intervenção terapêutica é dependente do quadro clínico da criança. Pacientes assintomáticos não requerem tratamento, optando-se por uma conduta expectante através de seguimento ambulatorial. Freqüientemente, a evolução é favorável e esses pacientes costumam ter atividade física normal durante toda a vida. Entretanto, algumas crianças, sobretudo quando atingem idade na qual se inicia a prática de exercícios, podem manifestar sintomas do tipo tonturas ou mesmo síncope, que indicam uma dificuldade de manutenção do débito sistêmico durante esforço. Nesses casos, deve ser considerada a possibilidade da implantação de marca-passo cardíaco ${ }^{16}$, assim como nas formas neonatais graves, associadas à miocardiopatia e relacionadas à presença de anticorpos maternos antiSSA/Ro e anti-SSB/LA.

TAQUICARDIA PAROXÍSTICA SUPRAVENTRICULAR (TPSV): habitualmente relacionada a uma imaturidade do sistema de condução intracardíaco ou, mais raramente, secundária à síndrome de Wolff-Parkinson-White (WPW), a TPSV é entidade relativamente freqüente no período neonatal ${ }^{17}$. Eventualmente, pode se manifestar associada a outras situações, como miocardite aguda e tumores intracardíacos. Os pacientes com esse tipo de arritmia costumam ser assintomáticos ou apresentam sintomas discretos e inespecíficos, como irritabilidade e gemência. Entretanto, alguns casos podem se manifestar com quadro de insuficiência cardíaca, particularmente aqueles com duração prolongada. Um dado importante, no exame físico, é que, freqüentemente, é o primeiro sinal da doença, é o nível de freqüência cardíaca, usualmente acima de $200 \mathrm{bpm}$, podendo, entretanto, atingir valores de 300 e até $400 \mathrm{bpm}$ em ritmo regular. A confirmação diagnóstica é habitualmente feita pelo eletrocardiograma convencional, devendo ser lembrada a necessidade de se estabelecer diagnóstico diferencial com o flutter atrial congênito, arritmia de ocorrência bastante rara. Esses pacientes necessitam internação em UTI pediátrica, com monitorização eletrocardiográfica contínua, em virtude da possibilidade (remota) de complicações e, também, para fazer a reversão da arritmia para ritmo sinusal após a terapêutica. Manobras vagais, como a compressão do seio carotídeo e imersão facial em água gelada, podem ser efetivas e devem ser tentadas com muito cuidado. Entretanto, a maioria dos pacientes requer terapia farmacológica para reversão da arritmia. En- tre as drogas habitualmente usadas encontram-se o sulfato de adenosina e a amiodarona entre as mais eficazes e que devem ser administradas endovenosamente. A digoxina deve ser usada com cautela, principalmente nos casos de síndrome de WPW e o verapamil é contra-indicado em neonatos, em virtude da possibilidade, já demonstrada, de induzir ao aparecimento de arritmias graves. O prognóstico dos casos de TPSV, secundários à imaturidade do sistema de condução, é bom, havendo desaparecimento gradual dos surtos de taquicardia. As formas associadas à síndrome de WPW necessitam de terapêutica invasiva, sendo usualmente empregada a ablação do feixe anômalo com radiofrequiência durante estudo eletrofisiológico. Outra possibilidade é a cirurgia cardíaca, essa atualmente de uso menos freqüente. Os resultados em longo prazo, após a terapêutica invasiva, costumam ser bons, sendo rara a ocorrência de recidiva da $\operatorname{arritmia}^{18}$ (Tabela V).

\section{Tabela V: Principais arritmias que reque rem terapêu uica ne onatal}

Taquicardia paroxistica supraventricular

\section{Bloqueio atrioventricular congerito completo}

Fintter atrial congêrito

Concluindo, acreditamos que a avaliação do RN com suspeita de cardiopatia deva ser realizada de maneira bastante elaborada e cuidadosa. O exame clínico cardiológico apurado é ainda elemento valioso, como bem enfatizado em publicação recente ${ }^{19}$, podendo fornecer informações cruciais para o diagnóstico. Usados rotineiramente nestes casos, o ECG e a radiografia de tórax costumam ser bastante úteis na investigação desses pacientes, que terão seu diagnóstico confirmado, na grande maioria dos casos, pelo ecocardiograma. As duas últimas décadas propiciaram um avanço considerável no tratamento das cardiopatias congênitas, estando disponíveis, hoje em dia, recursos farmacológicos e invasivos especificamente criados para tratamento dessas cardiopatias. Particularmente no período neonatal, os benefícios do cateterismo intervencionista e da cirurgia cardíaca pediátrica são notáveis, estando bem documentados. Entretanto, o uso racional desses recursos só será plenamente valorizado se a cardiopatia for precocemente suspeitada pelo clínico. 
Amaral F Granzotti JA, Manso PH \& Conti LS. When congenital heart disease should be suspected in the newborn. Medicina, Ribeirão Preto, 35:192-197, apr./june 2002.

ABSTRACT: Review article about the main clinical features of congenital heart disease presenting in the newborn period. Based on clinical data such as heart murmur, cyanosis, breathlessness and arrhythmia, the most frequently associated clinical findings are discussed as well as the entities most likely to be related to them. Informations on diagnosis and therapy are also briefly provided.

UNITERMS: Heart Defects, Congenital. Newborn. Heart Murmurs

\section{REFERÊNCIAS BIBLIOGRÁFICAS}

1 - SMYTHE JF; TEIXEIRA OHP VLAD P; DEMERS PP \& FELDMAN W. Initial evaluation of heart murmurs: are laboratory tests necessary? Pediatrics 86: 497-500, 1990.

2- AMARAL FTV; GRANZOTTI JA \& NUNES MA. Avaliação cardiológica em crianças com suspeita de cardiopatia. Resultados preliminares em 2000 pacientes. J Pediatr 71: 209213, 1995.

3- AMARAL FTV GRANZOTTI JA \& NUNES MA. Sopro cardíaco na criança. Experiência de um ambulatório especializado Rev Paul Pediatr 13 39-41, 1995.

4 - CHATELAIN P; OBERHANSLI I \& FRIEDLI B. Physiological pulmonary branch stenos is in newborns: 2Dechocardiographic and Doppler characteristics and follow up. Eur J Pediatr 152: 559-563, 1993.

5- GRAHAM Jr TP \& GUTGESSEL HP. Ventricular septal defects. In: MOSS AJ \& ADAMS EH, eds. Heart disease in infants, children and adolescents, including the fetus and young adult, 5 ed, Williams \& Wilkins, Baltimore, v. 1, p. 724746, 1995.

6- ROCCHINI AP \& EMMANOUILIDES GC. Pulmonary stenosis. In: MOSS AJ \& ADAMS EH, eds. Heart disease in infants, children and adolescents, including the fetus and young adult, 5 ed, Williams \& Wilkins, Baltimore, v. II, p. 930-962, 1995.

7 - FRIEDMAN WF. Aortic stenosis. In: MOSS AJ \& ADAMS EH, eds. Heart disease in infants, children and adolescents, including the fetus and young adult, 5 ed, Williams \& Wilkins, Baltimore, v. II, p. 1087-1111, 1995.

8 - BEEKMAN RH. Coarctation of the aorta. In: MOSS AJ \& ADAMS EH, eds. Heart disease in infants, children and adolescents, including the fetus and young adult, $5 \mathrm{ed}$, Williams \& Wilkins, Baltimore, v. II, p. 1111-1133, 1995.

9- GRAVES ED; REDMOND CR \& ARENSMAN RM. Persistent pulmonary hypertension in the neonate. Chest $93: 638-641$, 1988.

10- STEVENSON DK \& BENITZ WE. A practical approach to diagnosis and immediate care of the cyanotic neonate. Clin Pediatr 26: 325-331, 1987.
11 - KRAMER HH; SOMMER M, RAMMOS S \& KROGMANN O. Evaluation of $10 \mathrm{w}$ dose prostaglandin El treatment for ductus dependent congenital heart disease. Eur J Pediatr 154: 700-707, 1995

12 - FLYER DC, ROTHMAN KJ; BULKLEY LP; COHN HE; HELLENBRAND WE \& CASTANEDAA. The determinants of five year survival of infants with critical congenital heart disease. In: ENGLE MA, ed, Cardiovascular cunhes, FA Davis, Philadelphia, p. 393-405, 1981.

13- SALICE P; SEGANTINI A\& LOCATI E. The prognosis of premature atrial beats in infancy: a prospective study of 6900 infants (abstract). Circulation 68: 395, 1983. Suppl 3

14- JONES RWA; SHARP C; RABB LR; LAMBERT BR \& CHAM BERLAIN DA. 1028 neonatal electrocardiograms. Arch Dis Child 54: 427-431, 1979.

15 - MICHAELS M \& ENGLE MA. Congenital complete heart block: an international study of the natural history. Cardiovasc Clin 4: 85-101, 1972.

16- BERSTEINAD; CAMNAJ; FLETCHER R; GOLD RD; RICKARDS AF, SMYTH NPD; SPIELMAN SR \& SUTTON R. The NASPIBPEG generic pacemaker code for antibrady arrhythmia and adaptative-rate pacing and antitachycardia devices. Pacing Clin Electrophysiol 10: 794-799, 1987.

17- FISH F \& BENSON Jr DW. Disorder of cardiac rhythm and conduction. In: MOSS AJ \& ADAMS FH, eds. Heart dis ease in infants, children and adolescents, including the fetus and young adult, 5 ed, Williams \& Wilkins, Baltimore, v. II, p. 15551603, 1995.

18- TILL JA \& SI-IINEBOURNE EA. Supraventricular tachycardia diagnosis and management. Arch Dis Child 66: 647-652, 1991.

19- SILVA MAP. Reconhecimento das malformações cardíacas (cianose, insuficiência cardíaca, sopros e arritmias). In:

SANTANA MVT, ed. Cardiopatias congênitas no recém- nascido. Diagnóstico e tratamento, Atheneu, São Paulo, p. 89-101, 2000.

Recebido para publicação em 13/03/2002

Aprovado para publicação em 28/06/2002 